\title{
Aire Limitée Adaptation dynamique Développement InterNational - Limited Area Ensemble Forecasting (ALADIN-LAEF)
}

\author{
Martin Belluš ${ }^{1}$, Florian Weidle ${ }^{2}$, Christoph Wittmann ${ }^{2}$, Yong Wang ${ }^{2}$, Simona Taşku $^{3}$, and \\ Martina Tudor ${ }^{4}$ \\ ${ }^{1}$ Slovenský hydrometeorologický ústav, SHMU, Bratislava, Slovakia \\ ${ }^{2}$ Zentralanstalt für Meteorologie und Geodynamik, ZAMG, Vienna, Austria \\ ${ }^{3}$ Administratiei Nationale de Meteorologie, ANM, Bucharest, Romania \\ ${ }^{4}$ Državni hidrometeorološki zavod, DHMZ, Zagreb, Croatia
}

Correspondence: Martina Tudor (tudor@ cirus.dhz.hr)

Received: 7 December 2018 - Revised: 12 March 2019 - Accepted: 22 March 2019 - Published: 21 May 2019

\begin{abstract}
A meso-scale ensemble system Aire Limitée Adaptation dynamique Développement InterNational - Limited Area Ensemble Forecasting (ALADIN-LAEF) based on the limited area model ALADIN has been developed in the framework of Regional Cooperation for Limited Area modelling in Central Europe (RC LACE) consortium, focusing on short range probabilistic forecasts and profiting from advanced multi-scale ALARO physics. Its main purpose is to provide probabilistic forecast on daily basis for the national weather services of RC LACE partners. It also serves as a reliable source of probabilistic information applied to downstream hydrology and energy industry.
\end{abstract}

\section{Introduction}

There are a number of limited area model - ensemble prediction systems (LAM-EPS) in Europe. Here we describe the system developed in frame of Regional Cooperation for Limited Area modelling in Central Europe (RC LACE, Wang et al., 2018). Aire Limitée Adaptation dynamique Développement InterNational - Limited Area Ensemble Forecasting (ALADIN-LAEF) became operational in 2011, at that time having horizontal resolution of $18 \mathrm{~km}$ and 37 vertical levels (Wang et al., 2011). In 2013 the first substantial upgrade was made, incorporating the increase of horizontal and vertical resolutions to $11 \mathrm{~km}$ and 45 vertical levels, geographically bigger computational domain (Fig. 1) and a new ensemble of surface data assimilations involving perturbed screen-level observations (Belluš et al., 2016).

The ALADIN-LAEF system is going to higher resolution and finer scales. The horizontal and vertical resolutions are being increased to $5 \mathrm{~km}$ and 60 levels, respectively. As well as the use of higher resolution, several other upgrades are implemented, such as new model version, new physics parametrization schemes based on ALADIN System canonical model configuration ALARO (Termonia et al., 2018), additional stochastic perturbation of physics tendencies for the surface prognostic fields, etc. The ALARO CMC improvements are described in (Termonia et al., 2018) and this work focuses on the EPS improvement. The new ALADIN-LAEF system is defined on a slightly smaller domain (Fig. 1, red rectangle), that keeps the benefit over the global EPS (see analysis in Sect. 3), but will reduce the large increase of computational cost. A comparison of topography resolved by previous and upgraded system over the Alps and Tatra mountains is shown in Fig. 2. This topography is also the basis of the models' terrain following coordinate.

The ALADIN-LAEF system runs operationally on the High Performance Computer Facility at the European Centre for Medium-Range Weather Forecasts (ECMWF) twice a day with the integration starting at 00:00 and 12:00 UTC producing $72 \mathrm{~h}$ forecasts (Wang et al., 2010b). The ensemble consists of 1 unperturbed control run and 16 perturbed members involving initial condition uncertainty, model error 
Table 1. ALADIN-LAEF system specifications for current and new version.

\begin{tabular}{lll}
\hline ALADIN-LAEF & current & new \\
\hline Code version & cy36t1 & cy40t1 \\
Horizontal resolution & $10.9 \mathrm{~km}$ & $4.8 \mathrm{~km}$ \\
Vertical levels & 45 & 60 \\
Number of grid points & $500 \times 600$ & $750 \times 1250$ \\
Grid & quadratic & linear \\
Time step & $450 \mathrm{~s}$ & $180 \mathrm{~s}$ \\
Forecast length & $72 \mathrm{~h}(00: 00 / 12: 00$ UTC) & $72 \mathrm{~h}(00: 00 / 12: 00$ UTC) \\
Members & $16+1$ & $16+1$ \\
IC perturbation & ESDA [surface], breeding- & ESDA [surface], blending \\
& blending [upper-air] & (Phase I)/ENS BlendVar \\
& & (Phase II) [upper-air] \\
Model perturbation & ALARO-0 multi-physics & ALARO-1 multi-physics \\
& & + surface SPPT \\
LBC perturbation & ECMWF ENS & ECMWF ENS \\
SBUs consumed per year & $\sim 10$ mil & $\sim 120$ mil \\
\hline
\end{tabular}

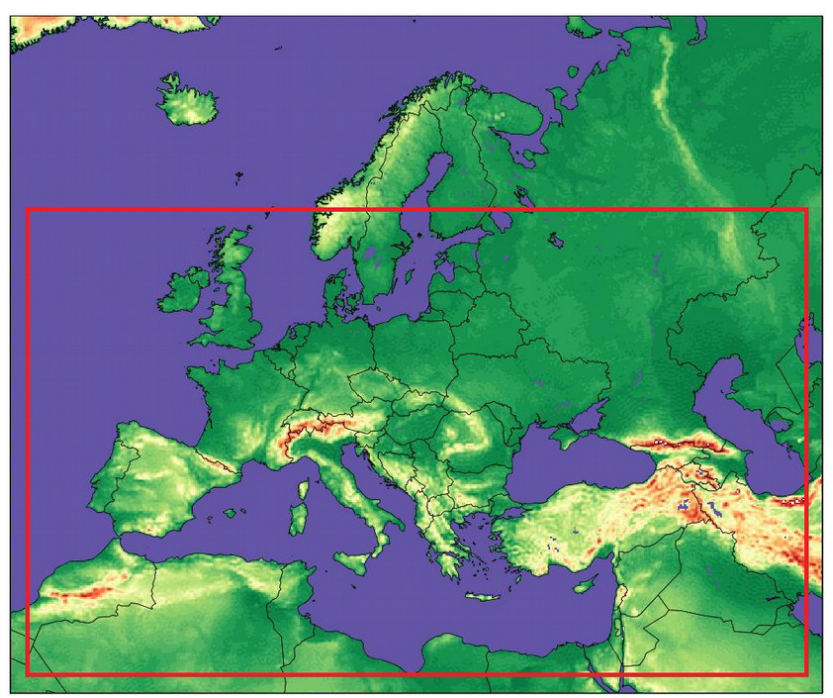

Figure 1. Current and new (red rectangle) ALADIN-LAEF computational domains.

simulation and coupling to perturbed lateral boundary conditions rendered by ECMWF EPS.

\section{Perturbation methods}

Together with modelling higher spatial resolutions the appropriate simulation of the uncertainties becomes more important. That is because the atmosphere naturally behaves chaotically at the smaller spatial and temporal scales. In the ALADIN-LAEF system we use different strategies to simulate the uncertainty of the initial conditions and of the numerical model, while the perturbations at the boundaries are prescribed by the downscaled information from driving global EPS.

\subsection{Initial condition perturbation}

The surface and soil prognostic fields' uncertainty in the initial conditions of ALADIN-LAEF system is simulated by the ensemble of surface data assimilations - ESDA (Belluš et al., 2016). This method replaced the former non-cycling surface breeding - NCSB (Wang et al., 2010a), which employed short-range surface forecasts driven by perturbed atmospheric forcing. The current ESDA profits from ALADIN surface data assimilation CANARI (Code d'Analyse Nécessaire à ARPEGE pour ses Rejets et son Initialisation), which is based on the Optimal Interpolation (OI) method. Each ensemble member has its own data assimilation cycle with randomly perturbed screen-level measurements (Fig. 3). The amplitude and direction of the perturbations are defined by a Gaussian distribution function with zero mean and standard deviation equal to the usual errors of the observations.

The uncertainty of the upper-air part of the initial conditions used in ALADIN-LAEF system is currently simulated by the breeding-blending cycle (Wang et al., 2014). It combines the large-scale perturbations provided by the driving global ensemble (ECMWF EPS), with the small-scale perturbations generated by ALADIN-LAEF breeding vectors within the pseudo-assimilation cycle. The upper-air spectral blending (Derková and Bellus, 2007) by digital filter initialization is used, profiting from the spectral character of ALADIN model. An obvious disadvantage of such method is the absence of data assimilation for the upper atmosphere. The ensemble BlendVar technique combines the ensemble of 3-D variational data assimilations (with perturbed observations like SYNOP, TEMP, AMDAR, GEOWIND and GNSS ZTD) and the upper-air spectral blending. Therefore, the implementation of the ENS BlendVar procedure is underway, to obtain more truthful control analysis and consequently the perturbed members with less initial bias. The novelty of ENS 

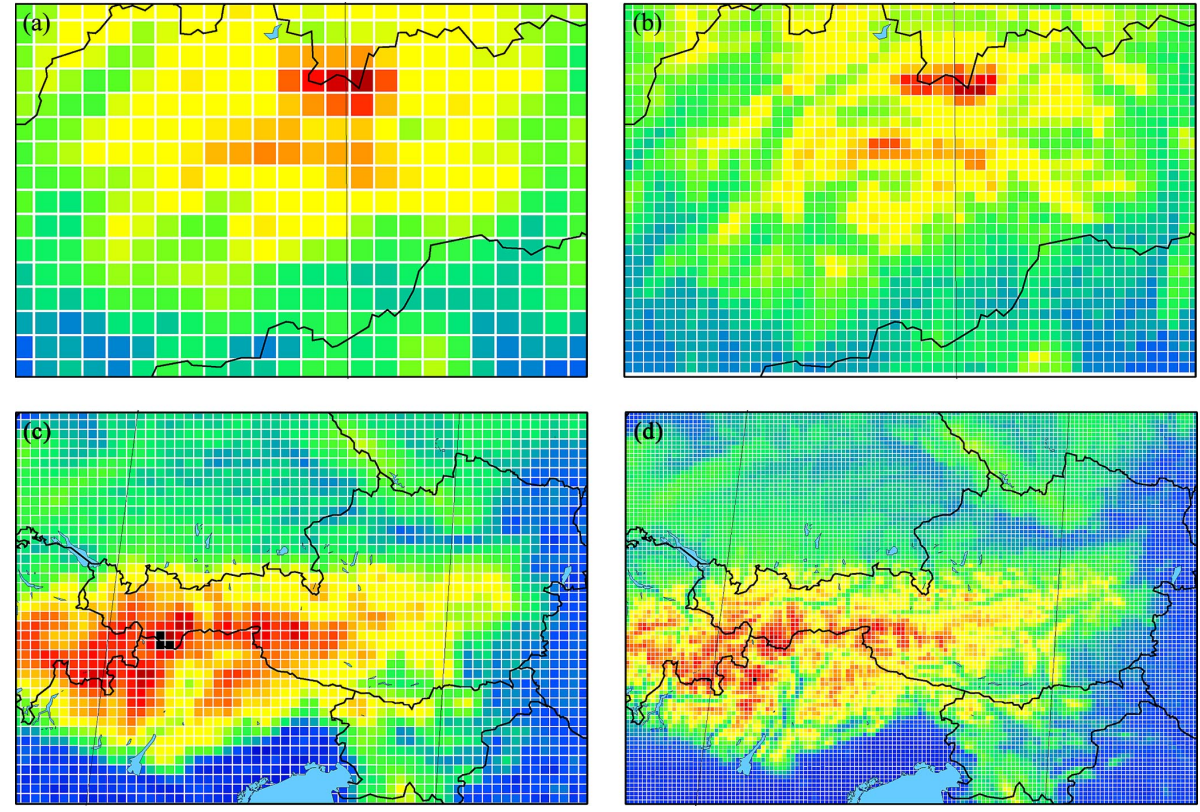

Figure 2. Model topography for current $11 \mathrm{~km}$ grid $(\mathbf{a}, \mathbf{c})$ and new $5 \mathrm{~km}$ resolution $(\mathbf{b}, \mathbf{d})$ zoomed over the Tatra mountains in northern part of Slovakia (a, b) and over the Austrian Alps (c, d).

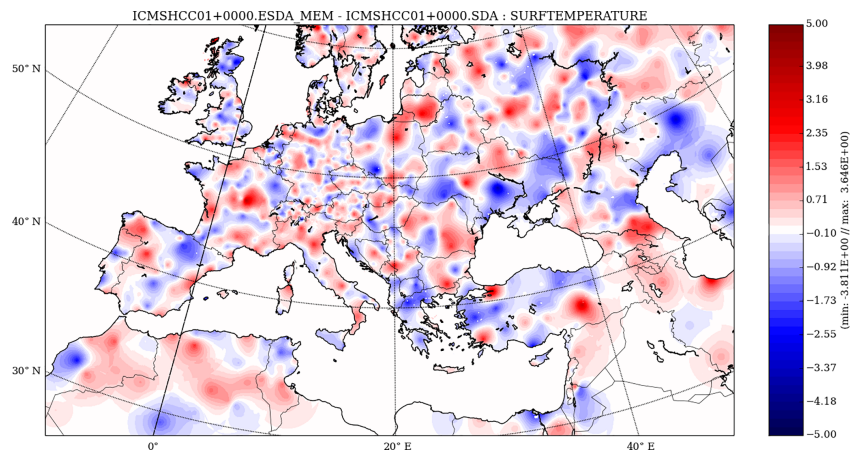

Figure 3. An example case of the Surface temperature perturbation by ESDA method (perturbed observation analysis minus reference analysis for one ensemble member).

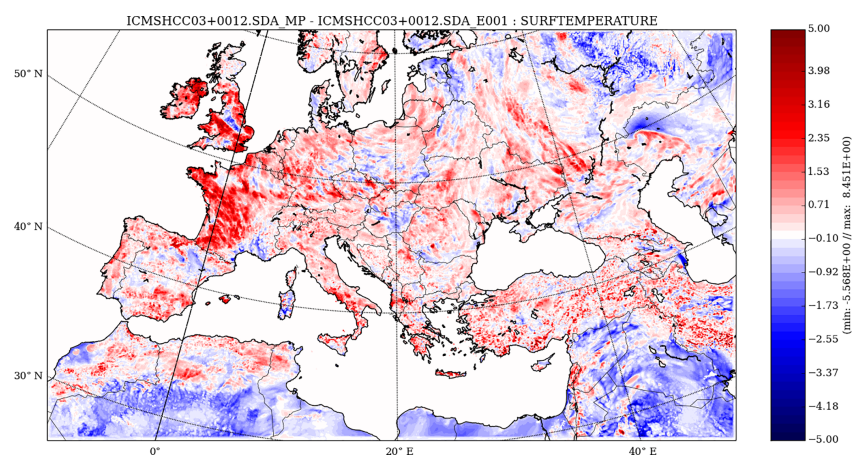

Figure 4. Surface temperature perturbation by multi-physics method (differences after $12 \mathrm{~h}$ of integration).

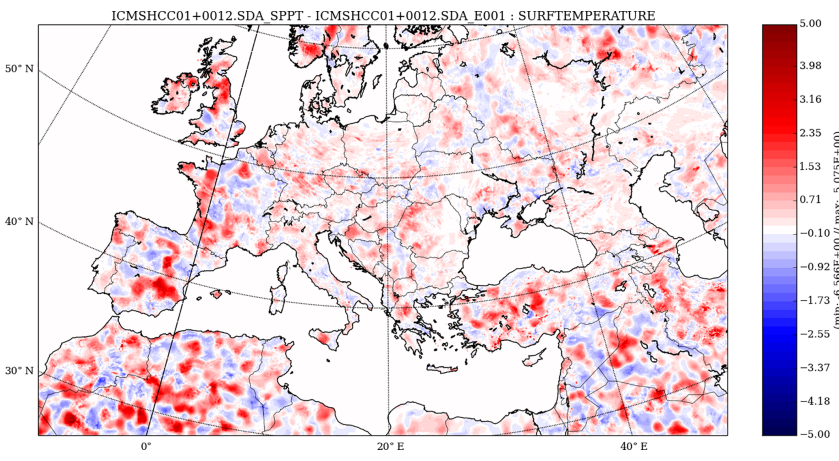

Figure 5. Surface temperature perturbation by stochastic physics method (differences after $12 \mathrm{~h}$ of integration).

BlendVar utilization within the LAM EPS is that it can serve as the method for generating the background error statistics (B-matrix) that can be used for operational 3D-Var in member services.

\subsection{Model perturbation}

During its lifetime, the meso-scale ensemble system ALADIN-LAEF has undergone many changes and upgrades. The forecast model uncertainty is currently simulated by several combinations of different micro-physics, deep and shallow convection, radiation and turbulence schemes. It largely profits from the multi-scale properties of the ALARO physics package used by the system (such as the modular multiscale microphysics and transport scheme for convection). 


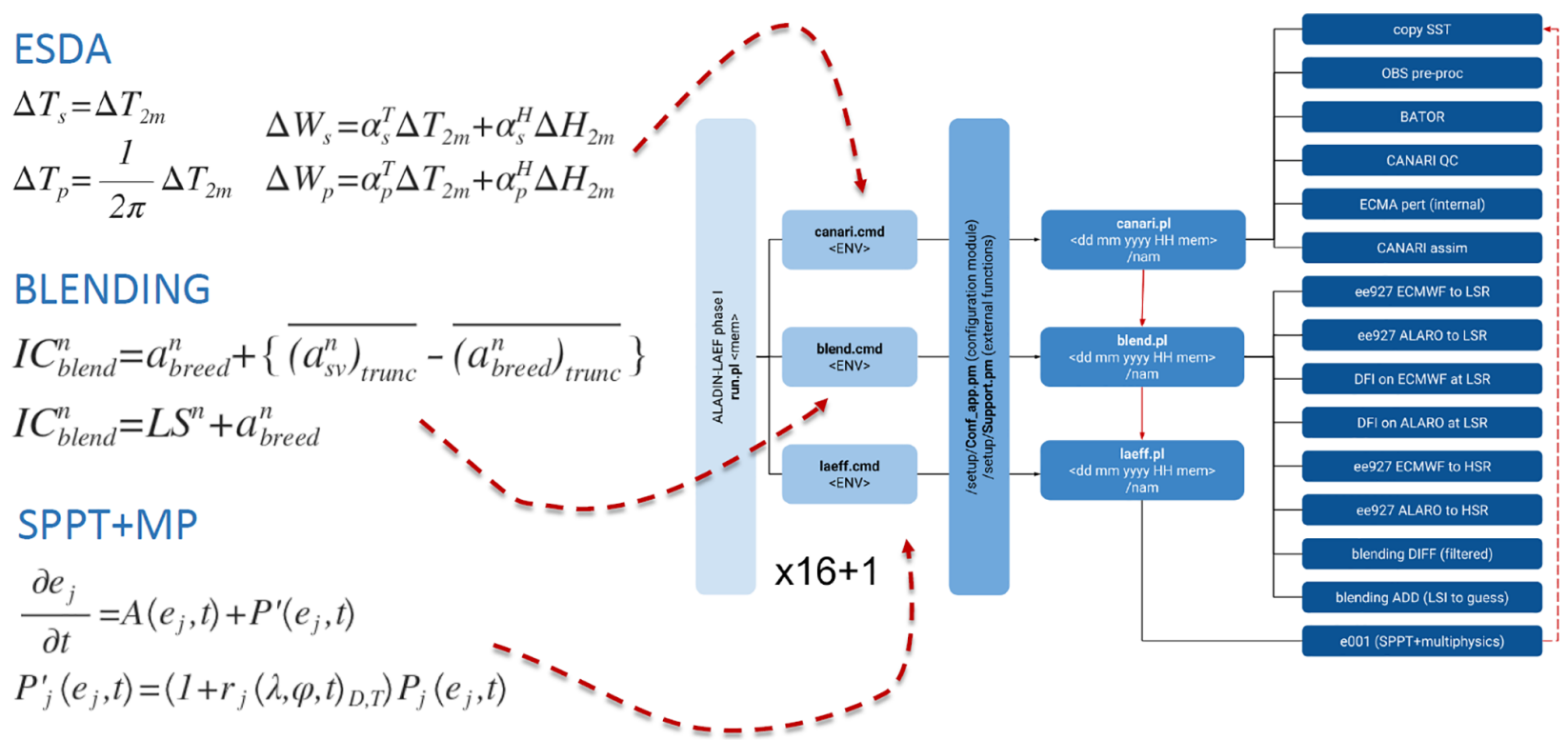

Figure 6. The scripting system of new ALADIN-LAEF Phase I configuration.
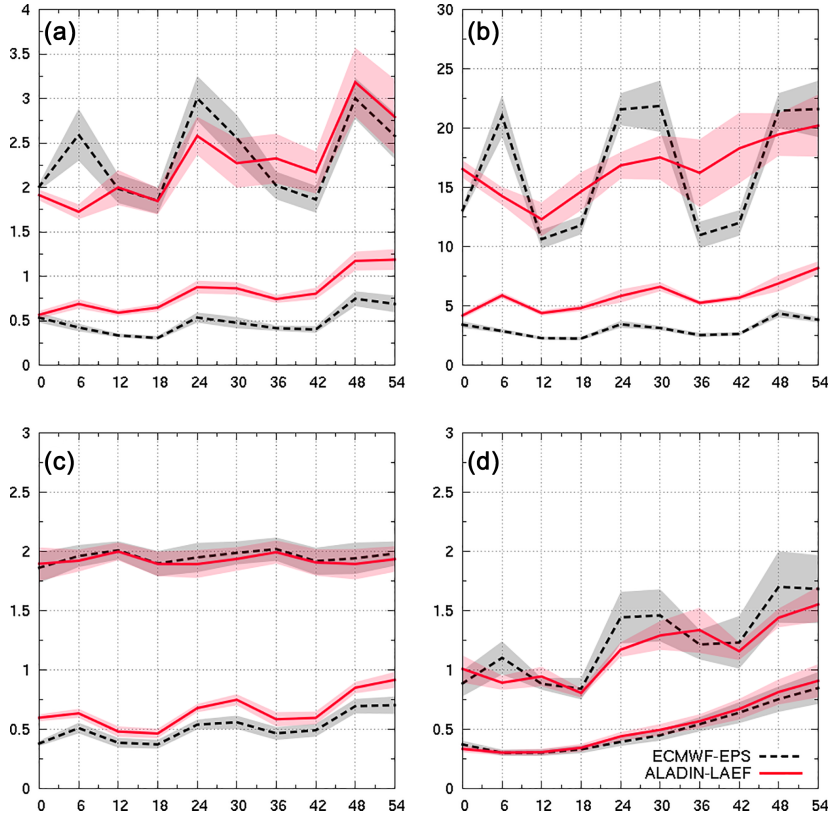

Figure 7. RMSE (upper lines) and spread of $2 \mathrm{~m}$ temperature (a), relative humidity (b), $10 \mathrm{~m}$ wind (c) and mean sea level pressure (d) versus forecast lead time, calculated against SYNOP observations for ECMWF ENS downscaling (black) and new ALADINLAEF Phase I (red). Shading denotes $10 \%$ and $90 \%$ confidence intervals.

The stochastic perturbation of physics tendencies (SPPT) is another approach which has been successfully tested in order to enhance the model uncertainty simulation for the surface prognostic variables, but also for the upper-air fields. The stochastic physics method can randomly disturb the model tendencies computed by the parametrization schemes and hence addresses the model accuracy at its source. In our experiments the surface prognostic fields like temperature, liquid soil water content, frozen soil water content, snow albedo, snow reservoir water content, snow density and water intercepted by vegetation were perturbed. We intentionally avoided the perturbation of deep soil prognostic fields (e.g. deep soil temperature), because such fields are naturally changing very slowly in time. On the other hand, we found the perturbation of (skin) surface prognostic fields very important for generating enough spread for screen-level variables in LAM EPS. The surface temperature is perturbed by assimilating perturbed screen-level measurements, while its effect is measured through the spread of the screen-level temperature forecast. Examples of surface temperature perturbations by multi-physics and SPPT are shown in Figs. 4 and 5. The multiphysics perturbation method yields dominantly positive or negative perturbations over the vast geographical areas, while the stochastic physics tendency perturbation yields small disturbances over the Central Europe in comparison to ESDA perturbations (for this example).

\subsection{Lateral boundary perturbation}

The ALADIN-LAEF system is driven by the global ECMWF EPS. The perturbed lateral boundary conditions (LBCs) are retrieved from the first 16 EPS members with a coupling frequency of $6 \mathrm{~h}$ to account for the uncertainties at the domain boundaries. This is a natural choice for the LBCs perturbation, not only because of the similarity in model physics and dynamics among the ECMWF IFS (Integrated Forecast System) and ALADIN, but also because of the quality of ECMWF forecasts and their operational availability. 

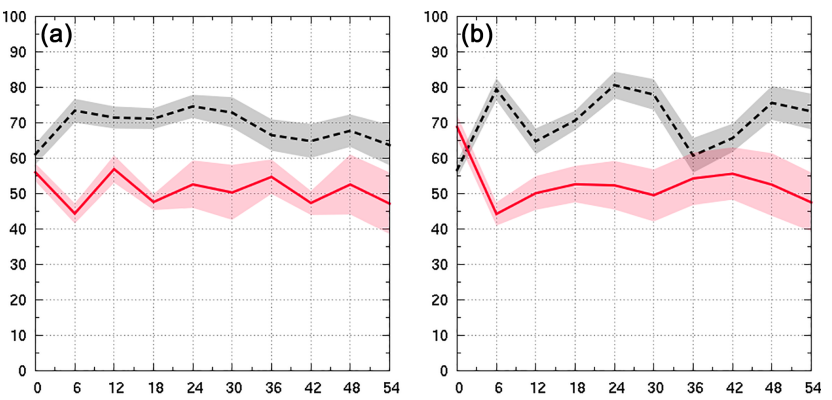

\subsection{Technical implementation}

Each of the 16 LAEF members (Fig. 7) uses different LBCs, different surface initial conditions from ESDA and different upper air initial conditions from blending. During the forecast, the surface fields are subject to SPPT and upper air fields are treated by multiphysics (4 different tunings of ALARO). The summary is given in Table 1 .

Higher spatial resolution and more complex and advanced physics has led to considerable increase in the computational cost of the new ALADIN-LAEF system.

The new ALADIN-LAEF suite (Fig. 6) uses a complex

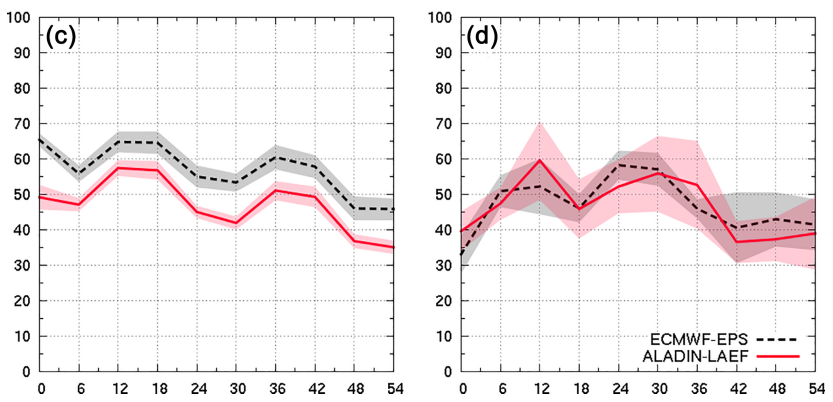

Figure 8. Outliers of $2 \mathrm{~m}$ temperature (a), relative humidity (b), $10 \mathrm{~m}$ wind (c) and mean sea level pressure (d) versus forecast lead time, calculated against SYNOP observations for ECMWF ENS downscaling (black) and new ALADIN-LAEF Phase I (red). Shading denotes $10 \%$ and $90 \%$ confidence intervals.
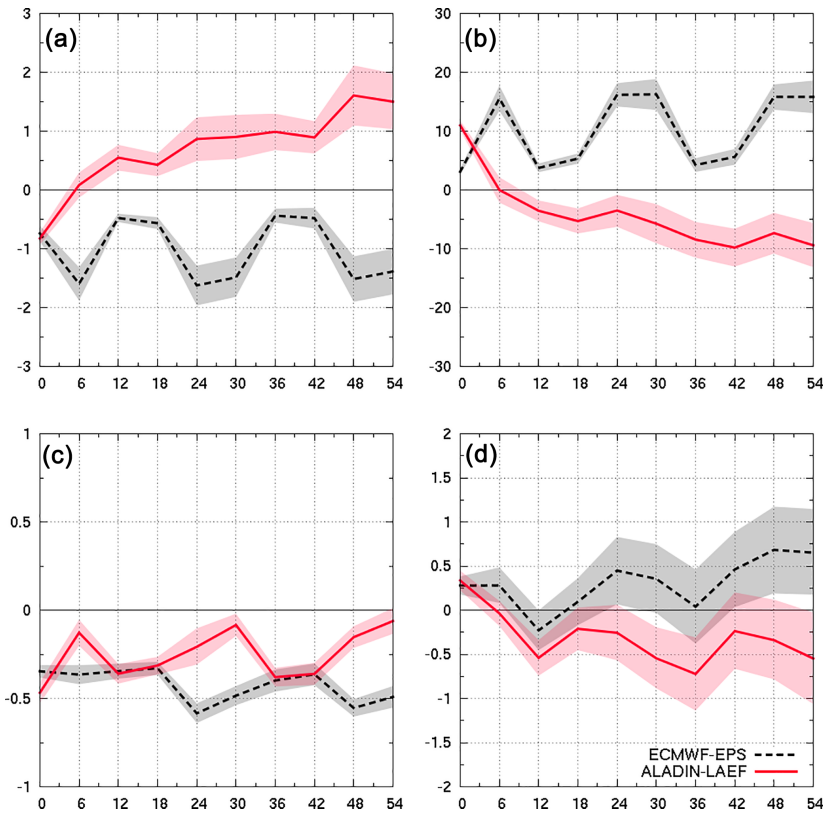

Figure 9. Bias of $2 \mathrm{~m}$ temperature (a), relative humidity (b), $10 \mathrm{~m}$ wind (c) and mean sea level pressure (d) versus forecast lead time, calculated against SYNOP observations for ECMWF ENS downscaling (black) and new ALADIN-LAEF Phase I (red). Shading denotes $10 \%$ and $90 \%$ confidence intervals.

\section{Results, validation and conclusions}

The main motivation for running an operational version of a regional EPS is its added value over the global ensemble. Therefore, we have verified new ALADIN-LAEF Phase I against the downscaling of corresponding 16 ECMWF EPS members for surface as well as for the upper-air parameters. For the surface parameters the statistical scores show larger spread (Fig. 7), lower root mean square error (RMSE), bias (Fig. 9) and a reduced number of outliers (Fig. 8) for new ALADIN-LAEF system compared to the downscaled ECMWF EPS. Another very positive result is also the significant damping of a diurnal cycle of errors, mostly for temperature and relative humidity. On the other hand, the impact on upper-air fields is rather neutral as it was expected (not shown).

Code and data availability. The ALADIN System code is not public. It is available for developers and researchers in national meteorological services members of LACE, ALADIN and HIRLAM consortia (and ECMWF). Please contact the corresponding author if the code or data are required.

Author contributions. MB, FW, CW and ST contributed to the development of ALADIN-LAEF System under the leadership of YW and subsequently MT. The manuscript was prepared by MB and MT. 
Competing interests. The authors declare that they have no conflict of interest.

Special issue statement. This article is part of the special issue "18th EMS Annual Meeting: European Conference for Applied Meteorology and Climatology 2018". It is a result of the EMS Annual Meeting: European Conference for Applied Meteorology and Climatology 2018, Budapest, Hungary, 3-7 September 2018.

Acknowledgements. We would like to express our gratitude to the many colleagues from RC LACE and ALADIN consortia who have contributed by their scientific work into the ALADIN-LAEF system, be it directly or even indirectly through the development of the ALADIN code. The running costs will be shared between several RC LACE partners who are also ECMWF Full Members (Austria, Croatia, Slovenia) and cooperating Turkey. The authors wish to acknowledge Turkey for the necessary System Billing Units (SBUs) as exchange for the operational probabilistic data and scientific cooperation. The authors also thank the anonymous reviewers for their valuable comments.

Review statement. This paper was edited by Emily Gleeson and reviewed by two anonymous referees.

\section{References}

Bellus, M., Wang, Y., and Meier, F.: Perturbing surface initial conditions in a regional ensemble prediction system, Mon. Weather Rev., 144, 3377-3390, 2016.

Derkova, M. and Bellus, M.: Various applications of the blending by digital filter technique in the ALADIN numerical weather prediction system, Meteorologicky casopis, 10, 27-36, 2007.
Termonia, P., Fischer, C., Bazile, E., Bouyssel, F., Brožková, R., Bénard, P., Bochenek, B., Degrauwe, D., Derková, M., El Khatib, R., Hamdi, R., Mašek, J., Pottier, P., Pristov, N., Seity, Y., Smolíková, P., Španiel, O., Tudor, M., Wang, Y., Wittmann, C., and Joly, A.: The ALADIN System and its canonical model configurations AROME CY41T1 and ALARO CY40T1, Geosci. Model Dev., 11, 257-281, https://doi.org/10.5194/gmd-11-2572018, 2018.

Wang, Y., Kann, A., Bellus, M., Pailleux, J., and Wittmann, C.: A strategy for perturbing surface initial conditions in LAMEPS, Atmos. Sci. Lett., 11, 108-113, 2010a.

Wang, Y., Bellus, M., Smet, G., and Weidle, F.: Use of ECMWF EPS for ALADIN-LAEF, ECMWF Newsletter, 126, Winter 2010/2011, 18-22, 2010b.

Wang, Y., Bellus, M., Wittmann, C., Steinheimer, M., Weidle, F., Kann, A., Ivatek-Šahdan, S., Tian, W., Ma, X., Tascu, S., and Bazile, E.: The Central European limited-area ensemble forecasting system: ALADIN-LAEF, Q. J. Roy. Meteor. Soc., 137, 483-502, 2011.

Wang, Y., Bellus, M., Geleyn, J.-F., Ma, X., Tian, W., and Weidle, F.: A new method for generating initial perturbations in regional ensemble prediction system: blending, Mon. Weather Rev., 142, 2043-2059, 2014.

Wang, Y., Bellus, M., Ehrlich, A., Mile, M., Pristov, N., Smolikova, P., Spaniel, O., Trojakova, A., Brozkova, R., Cedilnik, J., Klaric, D., Kovacic, T., Masek, J., Meier, F., Szintai, B., Tascu, S., Vivoda, J., Wastl, C., and Wittmann, C.: 27 years of Regional Co-operation for Limited Area Modelling in Central Europe (RC LACE), B. Am. Meteorol. Soc., 99, 1415-1432, 2018. 